\title{
An Integrated Powerpoint-Maple based Teaching-Learning Model for Multivariate Integral Calculus
}

\author{
Benchawan Wiwatanapataphee \\ Mahidol University \\ Sakda Noinang \\ Ubon Rachathani University \\ Yong Hong $\mathrm{Wu}$ \\ Curtin University of Technology Perth \\ Buraskorn Nuntadilok \\ Mahidol University
}

\begin{abstract}
In this paper, we present and evaluate a symbolic package based teaching-learning model for triple integral calculus. The model utilizes presentation and visualization technologies to assist in the teaching and learning of mathematical concepts and methods. The key mathematical concepts and integration techniques for triple integrals are introduced to students utilizing the animation feature of PowerPoint display supported by the graphic visualization function of the Maple software. To enhance students' learning process, a series of workbooks and symbolic tools are developed utilizing Maple 10 to guide students step-by-step in their studies. The developed symbolic tools also enable students to display the three-dimensional (3D) integration domain graphically and to check their calculation results. An investigation undertaken shows that with the proposed teaching-learning model, a higher percentage of students achieved a better understanding of the concepts and a better capacity in evaluating triple integrals with complicated integration domains, compared to traditional teaching and learning with no technology use. We purport that our technology model supports understanding by showing the 3D figures in integration from different angles, and also facilitates independent learning by reducing the need to draw these figures by the students themselves.
\end{abstract}

Keywords: symbolic package; integral calculus; Maple; graphic visualization

Over the last two decades, a number of symbolic packages for mathematics courses have been developed using MATHEMATICA, MATLAB or MAPLE. These symbolic packages have been used widely as tools in mathematics education. A computer algebra system (CAS) was used to teach double integration (Mathews, 1990), number theory (Cheung, 1996), graph sketching (Kong and Kwok, 1999), matrix multiplication (Cecil, 2002), mathematical analysis of electronic signals and circuits (Røyrvik, 2002). Recently, Cook (2006) developed a MAPLE graphing tool named calcIIIplots for 3D calculus teaching. Man (2007) introduced CAS Maple and its applications in mathematics education at school level. Students were provided with opportunities in using MAPLE to perform mathematics exploration or problem solving. Symbolic packages have also been used in teaching other subjects such as physics and engineering (Beltzer \& Shenkman, 1995; Johnson \& Buege, 1995; Ward, 2003) and computation (Tonkes, Loch, \& Stace, 2005). Tonkes et al. (2005) developed a learning model and designed a workbook for teaching computation using MATLAB to the first year students at the University of Queensland. Throughout the learning model, students work through the 
workbook to cover all topics to improve their proficiency in MATLAB. They found that the learning model has captured increased student support and has improved learning outcomes. The quality of student questions was improved and students displayed a deeper understanding of the mathematics.

Many researchers have measured and evaluated student learning of mathematics using symbolic packages (Kramarski \& Hirsch, 2003; Tall, 1991; Lagrange, 1999; Drijvers, 2000; Perjési, 2003; Heid, 1988; Kendal \& Stacey, 2002; Hayden \& Lamagna, 1998). It is found that symbolic packages enable students to achieve high level of logical analytical reasoning by visually supporting the concepts and the proofs with graphics presented through the packages (Fuchs, 2001; Drijvers, 2002; Peschek \& Schneider, 2002). Maple, based on a powerful scientific programming environments and libraries packages of special routines, is found to be one of the most popular systems because it is well-suited to aid college students to learn mathematics through verifying calculation and plotting complicated graphs, and also combines mathematical capabilities with a text editor.

Via the use of PowerPoint and Maple, we have developed an Integrated PowerPointMaple based (IPM) teaching-learning tool to aid in the teaching and learning of multivariate integral calculus, and an introduction of the tool has been published as a conference paper for the "Thirteenth Asian Technology Conference in Mathematics - 2008 (Noinang, Wiwatanapataphee, \& Wu, 2008)". In this paper, we present further details of the tool and investigate its effectiveness in improving the outcomes of teaching and learning. Briefly, the tool includes two parts: a teaching tool and a learning tool. The teaching tool consists of a series of PowerPoint slides integrated with Maple animations, and is designed for teachers to use in classroom teaching. The learning tool consists of a set of interactive Maplets and Maple worksheets. It is designed to enable students to have self-planned learning and selfassessment, and to reinforce students' conceptual understanding of integral calculus. The rest of the paper is organized as follows. First, the teaching model supported by symbolic calculation and graphic visualization of the teaching tool is presented. Afterwards, the Maple based step-by-step symbolic learning model using the learning tool is given. Evaluation of the teaching-learning model is then given and various maple procedures used in this paper are presented in Appendices.

\section{Powerpoint based Presentation with Computer Graphic Visualization Support}

Teaching triple integral calculus is a challenging task. In traditional mode of classroom teaching, students often find it difficult to understand instructors' explanations due to the difficulty in imagining the 3D integration domain. To overcome this problem, a teaching tool is developed utilizing the animation feature of PowerPoint display and the graphic visualization function of the Maple software.

For students to understand the concept of triple integrals and know how to evaluate triple integrals in different coordinate systems, the IPM teaching tool is designed to cover the following four essential components; (a) concepts of triple integrals; (b) evaluation of triple integrals in rectangular coordinates; (c) evaluation of triple integrals in cylindrical coordinates; (d) evaluation of triple integrals in spherical coordinates. 
For each of the components, the teaching tool consists of a number of PowerPoint slides and Maple graphs. The PowerPoint slides are used to explain the concepts and theories, while the Maple graphs are used to help students to visualize the concepts and methods graphically.

For the concept of triple integrals (Stewart, 2003), a PowerPoint slide is used to define the triple integral as a triple Riemann Sum, as shown in Figure 1a. Then, based on the definition, a number of examples are given to demonstrate the application of triple integrals. For example, if $f(x, y, z)$ is the material density of the object $\Omega$, then

$$
\iiint_{D} f(x, y, z) d \Omega=\lim _{l, m, n \rightarrow \infty} \sum_{i=1}^{l} \sum_{j=1}^{m} \sum_{k=1}^{n} f\left(x_{i j k}, y_{i j k}, z_{i j k}\right) \Delta V_{i j k}
$$

is the total mass of the object, as $f_{i j k} \Delta V_{i j k}$ is the approximation of the mass of one of the subboxes of $\Omega$ and the summations are to add the mass of all the sub-boxes in $\Omega$ together. The concept of triple integral, as a triple Riemann sum, can be clearly illustrated graphically by the Maple graphic visualization function as shown in Figure $1 b$.

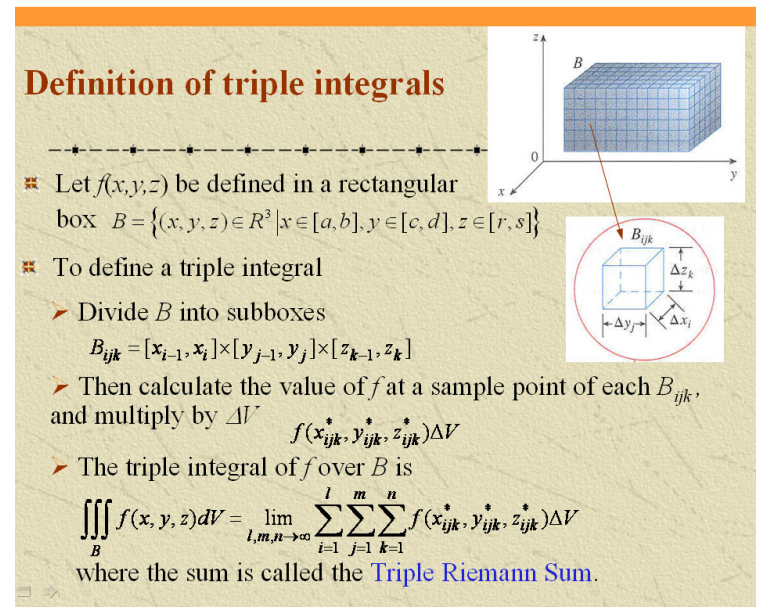

Figure 1a. The concept of triple integral Mathematical definition of triple integral.

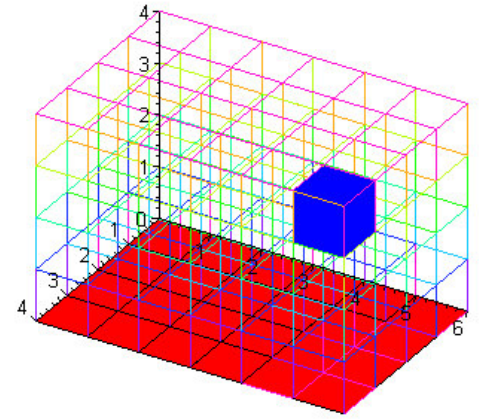

Figure $1 b$. The concept of triple integral - Maple animation showing the concept of triple Riemann sum.

To study the method for evaluation of triple integrals over a general domain in rectangular coordinates, we first use Maple graphic functions to demonstrate that integration domains in rectangular coordinates can be classified into $x$-cap region, $y$-cap region, $z$-cap region or combination of them (Wu \& Wiwatanapataphee, 2008). Then, we use PowerPoint slides to describe how to evaluate triple integrals over each type of the regions. As an example, Figure 2 gives the PowerPoint slide and the Maple graph used for demonstrating how to evaluate a triple integral over a $z$-cap region. In the classroom lecture presentation, firstly, the instructor clicks on Figure $2 a$ to start up the animation of the object in Figure $2 b$ demonstrating graphically that a $z$-cap region is a region bounded by an upper surface $z=z_{2}(x, y)$ and a lower surface $z=z_{1}(x, y)$ with a projection $D$ on the $x y$-plane. Then, through the PowerPoint slide, the instructor shows that a $z$-cap region $E$ can be visualized graphically as a set of points $(x, y, z)$ for which $(x, y)$ takes values in $D$ and for each point ( $x$, $y$ ) in $D, z$ changes from $z=z_{1}(x, y)$ to $z=z_{2}(x, y)$ and consequently the evaluation of a triple 
integral over a $z$-cap region $E$ becomes first evaluating a single integral with respect to $z$ from $z_{1}(x, y)$ to $z_{2}(x, y)$ and then evaluating a double integral with respect to $x$ and $y$ over $D$. The graphic visualization helps to understand the integration procedure.

(2) Over z-cap (type 1) region (a solid between 2 z-caps):

$E=\left\{(x, y, z)(x, y) \in D, u_{1}(x, y) \leq z \leq u_{2}(x, y)\right\}$

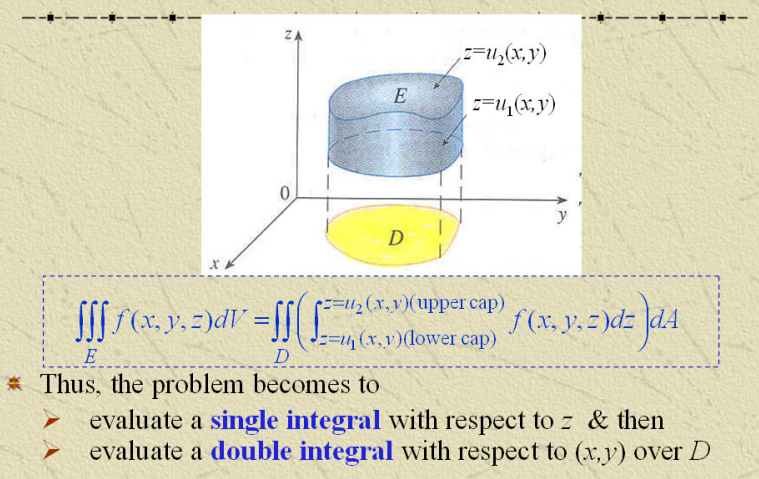

Figure 2a. Evaluation of triple integrals over a $z$ cap region - A PowerPoint slide showing the method of evaluation.

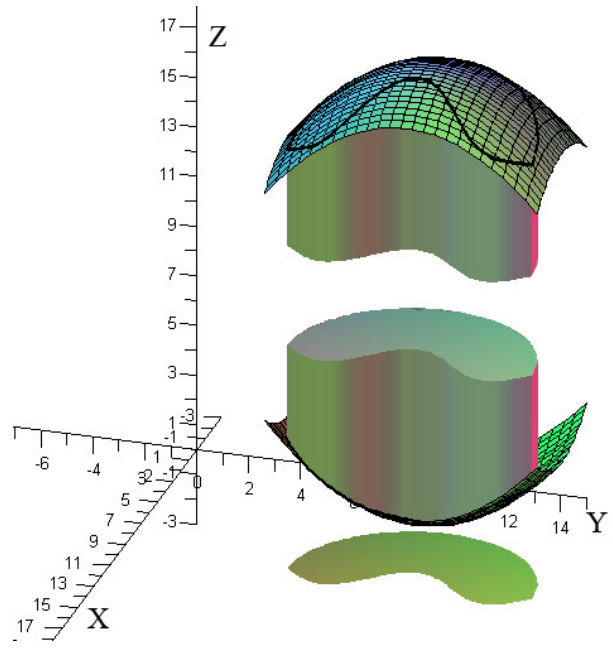

Figure $2 b$. Evaluation of triple integrals over a $z$-cap region - Maple animation showing the concept of a $z$-cap region.

For evaluating triple integrals in cylindrical coordinates, first, we use the Maple graphic function to demonstrate graphically that a point in space can be identified by the cylindrical polar coordinates $(r, \theta, z)$ representing the intersection of the cylindrical surface of radius $r$, the vertical plane with angle $\theta$ from the $x z$-plane and the horizontal plane with distance $z$ from the $x y$-plane, as shown in Figure 3a. Then, from the figure, we show on the PowerPoint slide how to relate the $(r, \theta, z)$ coordinates with the $x, y$, and $z$ coordinates, and then, we use the Maple graphic function to demonstrate that a differential element (as shown in Figure $3 b$ ) can be obtained by cutting the object with two cylindrical surfaces $(r=r, r=r+\Delta r)$ and two $z$-planes $(z=z, z=z+\Delta z)$ as well as two $\theta$-planes $(\theta=\theta, \theta=\theta+\Delta \theta)$. Consequently, the volume of the differential element can be calculated from its geometry as follows

$$
d V=A_{x y} \times d z=\left[(r+d r)^{2} d \theta / 2-r^{2} d \theta / 2\right] \times d z=\left(r+\frac{1}{2} d r\right) d r d \theta d z,
$$

where $A_{x y}$ is the area of the projection of the differential element on the $x y$-plane. As $d r$ is infinitesimal, $r+\frac{d r}{2} \rightarrow r$ and thus $d V=r d r d \theta d z$. 


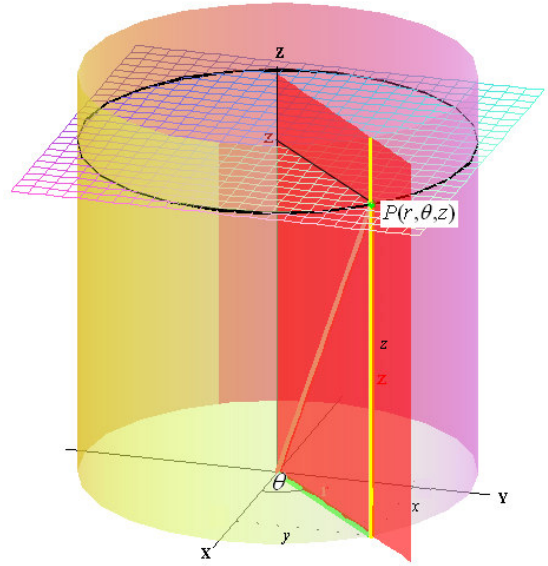

Figure 3a. Cylindrical coordinates - A point in space can be identified by $P(r, \theta, z)$ where $x=r \cos \theta, y=r \sin \theta$.

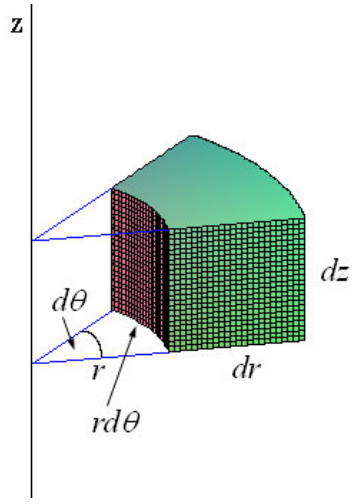

Figure $3 b$. Differential element - The volume of the differential element is $d V=r d z d r d \theta$.

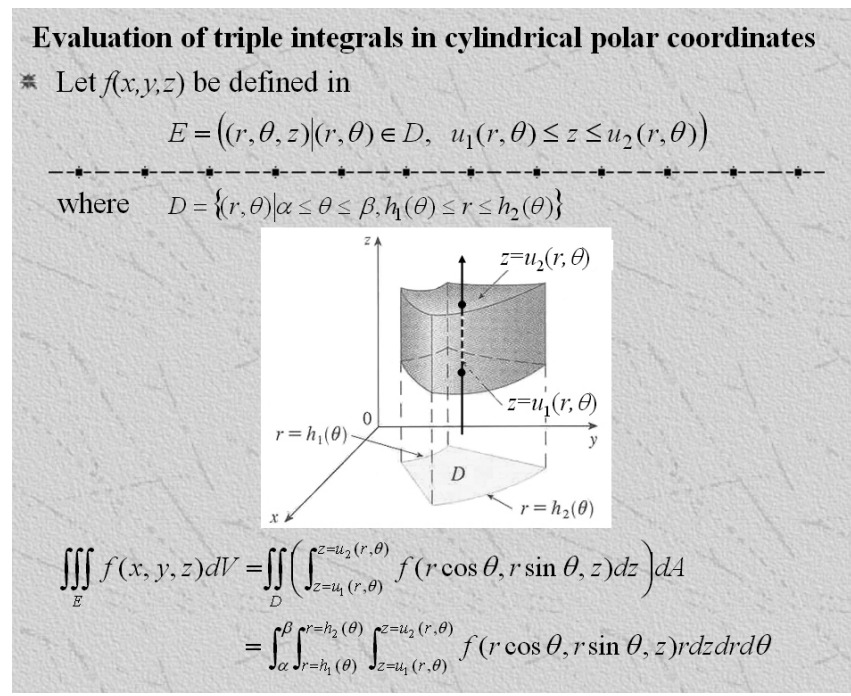

Figure 4. PowerPoint slide demonstrating how to evaluate a triple integral in cylindrical coordinates.

Finally, we demonstrate that a domain as shown in Figure 4, defined in cylindrical polar coordinates, can be visualized as a $z$-cap region and thus the integration over the region becomes evaluating a single integral with respect to $z$ firstly from $z=u_{1}(r, \theta)$ to $z=u_{2}(r, \theta)$ and then evaluating a double integral with respect to $r$ and $\theta$ over the region $D$ in polar coordinates.

Similarly, for evaluating triple integrals in spherical polar coordinates, we first use the Maple graphic function to demonstrate that a point in space can be identified by a spherical polar coordinates $(\rho, \theta, \phi)$ representing the intersection of the spherical surface with radius $\rho$ and centre at the origin, the vertical plane with angle $\theta$ from the $x z$-plane, and a cone surface with angle $\phi$ with the $z$-axis, as shown in Figure 5a. Then from the graph, we derive the relation between the $x, y$, and $z$ coordinates and the $\rho, \theta$, and $\phi$ coordinates, and then, 
we use the Maple graphic function to demonstrate that a differential element (as shown in Figure $5 b)$ can be obtained by cutting the object with two spherical surfaces $(\rho=\rho$, $\rho=\rho+d \rho)$ and two cone surfaces $(\phi=\phi, \phi=\phi+d \phi)$ as well as two $\theta$-planes $(\theta=\theta$, $\theta=\theta+d \theta$ ). We then proceed to calculate the volume of the differential element by utilizing the formula for the volume of spherical cone

$$
V(\rho, \phi)=\frac{2}{3} \pi \rho^{3}(1-\cos \phi),
$$

where $\rho$ is the radius of the sphere and $\phi$ is half of the cone angle as shown in Figure 7.

Let $V_{1}$ be the volume of the solid cut by the two spherical surfaces ( $\rho=\rho$ and $\rho=\rho+d \rho)$ within the cone $\phi=\phi+d \phi$, then

$$
V_{1}=V(\rho+d \rho, \phi+d \phi)-V(\rho, \phi+d \phi) .
$$

Similarly, the volume of the solid cut by the two spherical surfaces within the cone $\phi=\phi$ is

$$
V_{2}=V(\rho+d \rho, \phi)-V(\rho, \phi) .
$$

Hence, the volume of the solid cut by the two spherical surfaces and the two cone surfaces is $V_{1}-V_{2}$ and the volume of the differential element is

$$
\begin{aligned}
& d V=\frac{d \theta}{2 \pi}\left(V_{1}-V_{2}\right) \\
& \quad=\frac{d \theta}{2 \pi}\{[V(\rho+d \rho, \phi+d \phi)-V(\rho, \phi+d \phi)]-[V(\rho+d \rho, \phi)-V(\rho, \phi)]\} .
\end{aligned}
$$

Using the Taylor series approximation to the first order, we have

$$
\begin{aligned}
d V & =\frac{d \theta}{2 \pi}\left\{\frac{\partial V}{\partial \rho}(\rho, \phi+d \phi) d \rho-\frac{\partial V}{\partial \rho}(\rho, \phi) d \rho\right\} \\
& =\frac{d \theta}{2 \pi}\left\{\frac{\partial^{2} V(\rho, \phi)}{\partial \rho \partial \phi} d \rho d \phi\right\} \\
& =\frac{d \theta}{2 \pi} 2 \pi \rho^{2} \sin \phi d \rho d \phi \\
& =\rho^{2} \sin \phi d \rho d \theta d \phi
\end{aligned}
$$

We should also address here that the lengths in the $r, \phi$ and $\theta$ directions of the element are respectively $d \rho, \rho d \phi$ and $\rho \sin \phi d \theta$ (as shown in Figure 5b) and the volume of the element is the same as that of a rectangular box with dimension $d \rho \times \rho d \phi \times \rho \sin \phi d \theta$. Finally, in the PowerPoint slide (Figure 6), the formula for evaluation of a triple integral in spherical coordinates is given. 


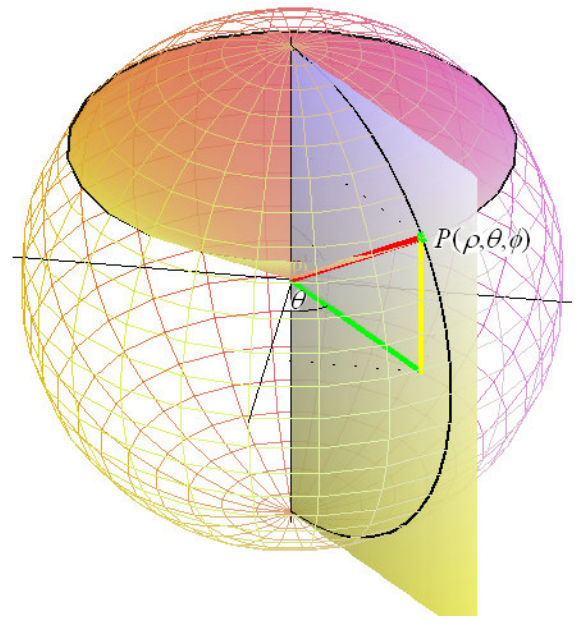

Figure 5a. Spherical coordinates - A point in space can be identified by $P(\rho, \theta, \phi)$ where $x=\rho \sin \phi \cos \theta, \quad y=\rho \sin \phi \sin \theta$, $z=\rho \cos \phi$.

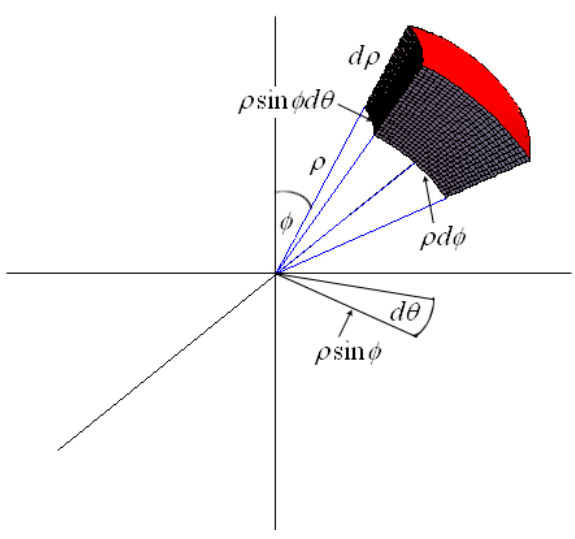

Figure 5b. Differential element and its volume - The volume of the differential element is $d V=\rho^{2} \sin \phi d \rho d \theta d \phi$.

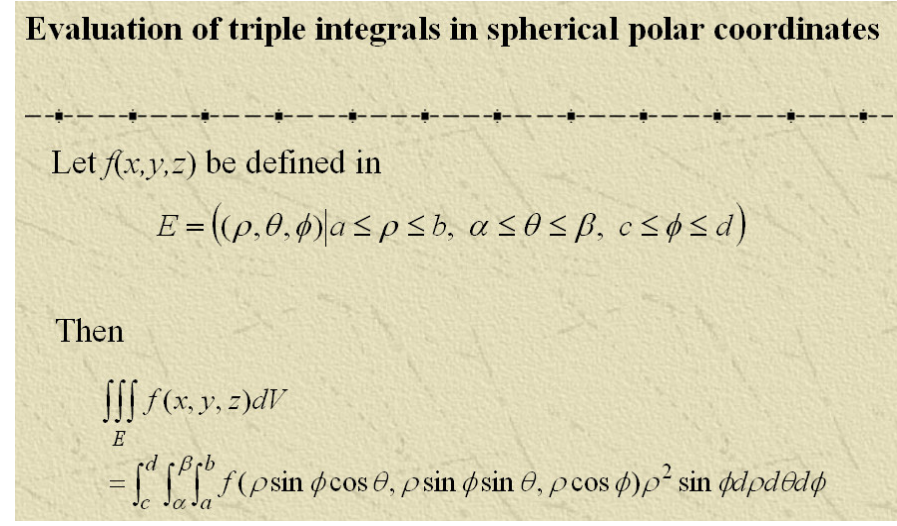

Figure 6. PowerPoint slide showing how to evaluate triple integrals in spherical coordinates.

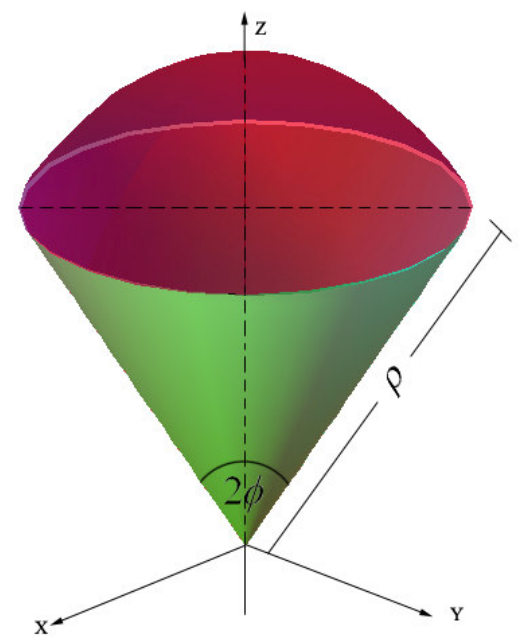

Figure 7. A spherical cone with radius $\rho$ and cone angle $2 \phi$. 


\section{Maple Based Step-by-Step Symbolic Learning Tool}

For most students, the main difficulty in the study of triple integral calculus is the sketching of integration domain and the subsequent determination of integration bounds (Cook 2006, Wu \& Wiwatanapataphee, 2008). To enhance students' self-learning capability, an interactive symbolic learning tool based on Maple is developed for the triple integral calculus. The tool, a Maplet, allows students to interact with computer and solve problems step by step. Basically, the Maplet has three basic functions: (a) display the graphs of functions; (b) define the integral; (c) evaluate the integral and show the symbolic result in an output window. Obviously, the graphic display is important as it helps students to graphically visualize the integration domain bounded by general surfaces defined by functions, so as to determine whether the domain is a $z$-cap region, $y$-cap region, or $x$-cap region and consequently determine the integration order and integration bounds for the definition of the problem. The following gives an example.

\section{Example}

Find the mass of the solid with constant mass density $\rho(x, y, z)=1$ bounded by a cylindrical surface $x^{2}+y^{2}=16$ and three planes: $y=3, z-y=3, z+2 y=-3$.

\section{Solution}

Firstly, in the input window, enter the equations for the cylindrical surface and the three planes, and also enter the display range in the $x, y$ and $z$ directions. Then, click on the plot button to view the surface and planes and their interfaces as shown in Figure 8. Obviously, this is a $z$-cap region bounded by a lower $z$-surface $z=-3-2 y$ and an upper $z$-surface $z=3+y$ with a projection $D$ on the $x y$-plane. To determine the bounds of integration in the $x$ and $y$ directions, project the integration domain onto the $x y$-plane as shown in Figure 9. Obviously,

$$
D=\left\{(x, y) \mid-\sqrt{16-y^{2}} \leq x \leq \sqrt{16-y^{2}},-2 \leq y \leq 3\right\} .
$$

Hence,

$$
m=\iint_{D}\left[\int_{-3-2 y}^{3+y} \rho d z\right] d A=\int_{-2}^{3} \int_{-\sqrt{16-y^{2}}}^{\sqrt{16-y^{2}}} \int_{-3-2 y}^{3+y} d z d x d y .
$$

To evaluate the above integral using Maplet, firstly choose the order of integration to be " $d z d x d y$ ", then input the bounds of integration, then double click on the "Show integration region" button to display the region of integration and then click on the "Evaluate the integral" button to evaluate the integral for the mass $m$ step-by-step on the result window, yielding $m=72 \sqrt{3}+16 \pi+4 \sqrt{7}+96 \arcsin \frac{3}{4}$ as shown in Figure 10 . 


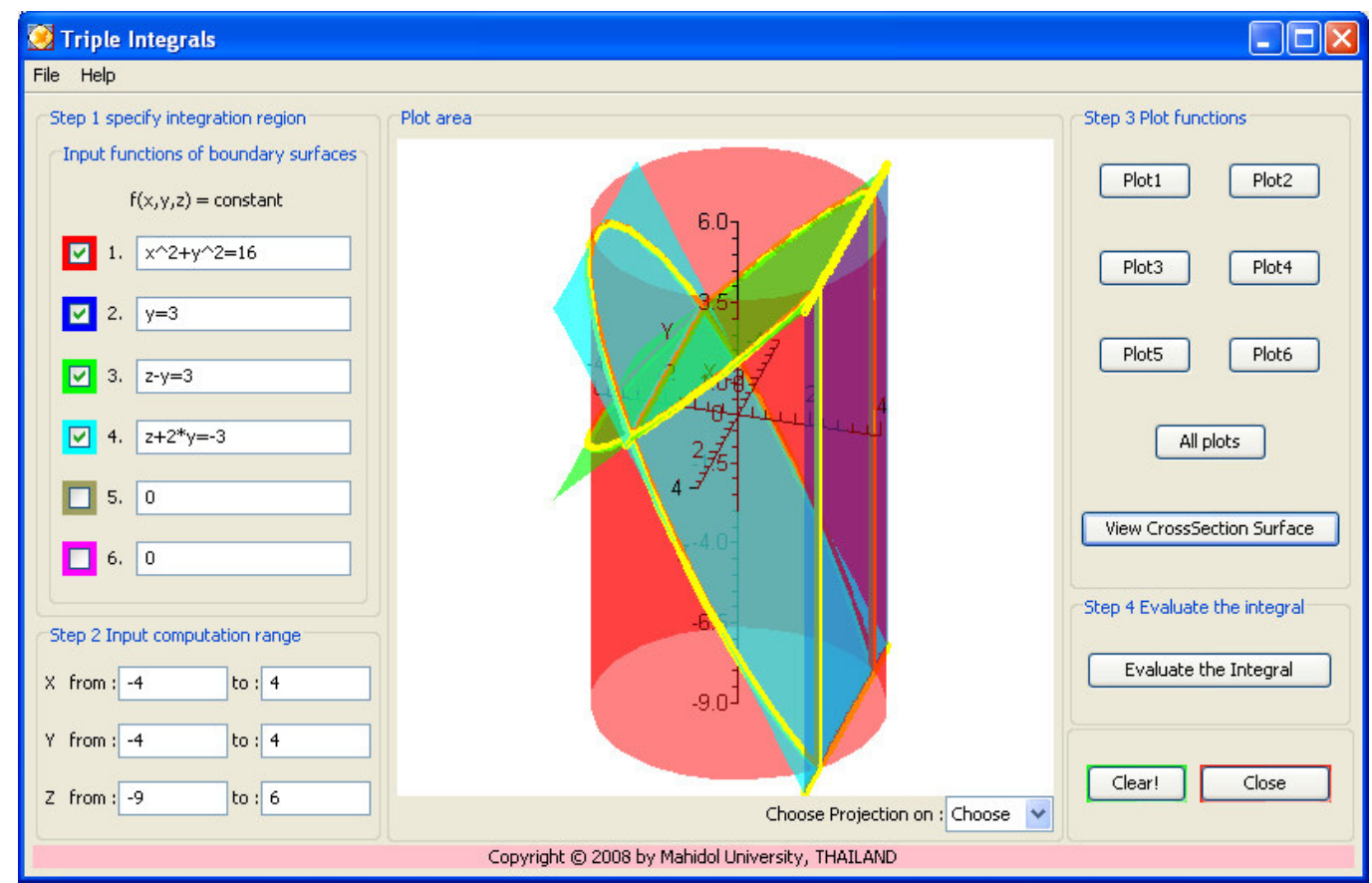

Figure 8. Maplet window showing the boundary surface: $x^{2}+y^{2}=16, y=3, z-y=3$, and $z+2 y=-3$, and their intersections.

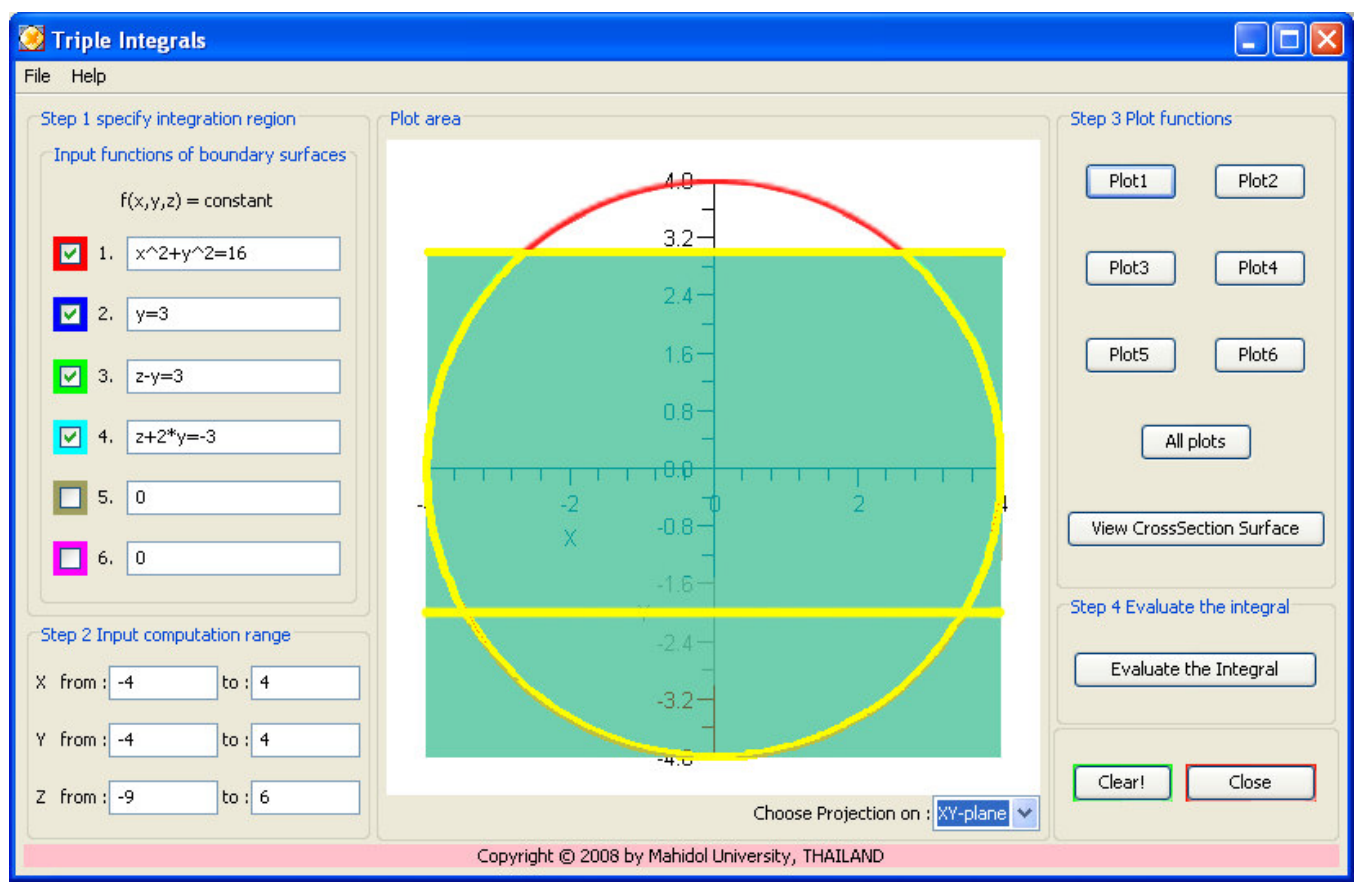

Figure 9. Maplet window showing the projection of the cylinder and 3 planes as defined in Step 1 on the $x y$-plane. 


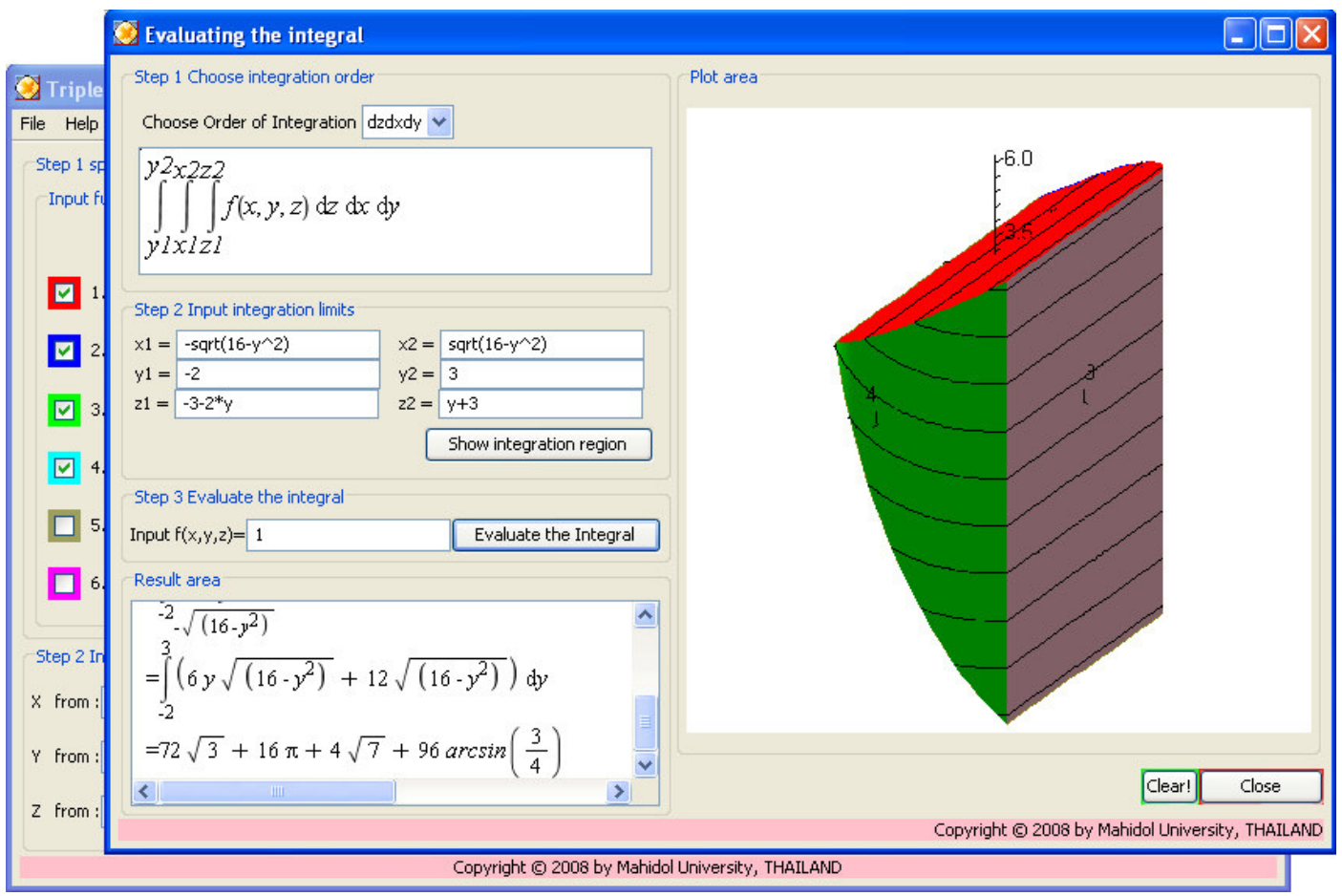

Figure 10. Maplet window showing the 3 steps for evaluating the integral and the result.

\section{Investigating the Effectiveness of the IPM Based Teaching-Learning Model}

In general, mathematics is considered, within the community of students and social in general, as a hard subject, and triple integral calculus is particularly the case. With the aim of making the integral calculus simple, interesting and attractive, a teaching-learning model supported by symbolic calculation and graphic visualization has been developed and presented in the previous two sections.

To evaluate the effectiveness of the developed teaching-learning model in improving students' learning outcomes, an investigation was undertaken at Kasetsart University Chalermphrakiat Sakonnakhon Province Campus involving two teachers, one observer and 73 engineering students. The students were divided into two groups: a computer-aided group (IPM) consisting of 32 students, and a traditional teaching-learning (TTL) group with 41 students. Firstly, students in both groups were asked to sit in a pre-test. The pre-test consists of 35 multiple-choice questions, tapping into conceptual and procedural aspects of the 3D integration. For each question, four answers are given including one correct answer and three incorrect answers. If the correct answer is chosen for a question, the student receives a score of one for the

question or otherwise zero. Thus, the maximum score available for the pre-test is 35 . After the test, students in the IPM group were taught the triple integral by using the IPM teachinglearning model, while the other group was taught by using the traditional teaching learning model. Then, we conducted a post-test. The post-test also consists of 35 multiple-choice questions with a total core of 35 . The following is an example question 
Question 1. $\int_{0}^{1} \int_{0}^{1-x} \int_{0}^{1-x-y} f(x, y, z) d z d y d x=$
(a) $\int_{0}^{1} \int_{0}^{1-x-y} \int_{0}^{1-x} f(x, y, z) d y d z d x$
(b) $\int_{0}^{1} \int_{0}^{1-z} \int_{0}^{1-y-z} f(x, y, z) d x d y d z$
(c) $\int_{0}^{1} \int_{0}^{1-x} \int_{0}^{1-y-z} f(x, y, z) d x d y d z$
(d) $\int_{0}^{1} \int_{0}^{1-x} \int_{0}^{1-y-z} f(x, y, z) d x d z d y$

The results obtained by both groups in the pre-test and post-test are summarized in Table 1 .

Table 1

Scores obtained by the two experiment groups in the pre-test and the post-test

\begin{tabular}{lrrrr}
\hline & \multicolumn{2}{c}{ IPM group } & TTL group \\
& Mean & SD & Mean & SD \\
\hline Pre-test & 8.50 & 2.29 & 9.39 & 2.06 \\
Post-test & 21.47 & 4.45 & 18.85 & 4.07 \\
\hline
\end{tabular}

It is clear from the results that students in the integrated PowerPoint Maple (IPM) group achieved higher mean score than those in the TTL group.

Table 2

One-way ANOVA for pre-test scores

\begin{tabular}{lccccc}
\hline & $\begin{array}{c}\text { Sum of } \\
\text { Squares }\end{array}$ & df & $\begin{array}{c}\text { Mean } \\
\text { square }\end{array}$ & F & sig. \\
\hline Between groups & 14.244 & 1 & 14.244 & 3.048 & .085 \\
Within groups & 331.756 & 71 & 4.673 & & \\
Total & 346.000 & 72 & & & \\
\hline
\end{tabular}

Table 3

One-way ANOVA for post-test scores

\begin{tabular}{lccccc}
\hline & $\begin{array}{c}\text { Sum of } \\
\text { squares }\end{array}$ & df & $\begin{array}{c}\text { Mean } \\
\text { square }\end{array}$ & F & sig. \\
\hline Between groups & 122.909 & 1 & 122.909 & 6.844 & .011 \\
Within groups & 1275.091 & 71 & 17.959 & & \\
Total & 1398.000 & 72 & & & \\
\hline
\end{tabular}

To determine whether the difference of the mean scores between the two groups is due to group membership (effect of teaching-learning model) or others, the one-way ANOVA variance analysis (Peck, 2008) is carried out. Table 2 and Table 3 shows the ANOVA for pretest scores and post-test scores, respectively. For $\alpha=0.05, F_{\text {critical }}(0.05,1,71)=3.9863$. Hence from the data in the Tables, it is clear that the mean scores between the groups for the pre-test 
are not significantly different, while the mean scores of the IPM group for the post-test is significantly higher than that of the other group.

We also undertook an open-ended student survey to get students' opinions on the IPM teaching-learning model. In the survey, students were asked whether the proposed teachinglearning model helped them to have a better understanding of the mathematical concepts and facilitated their learning of the methods for the evaluation of multivariate integral calculus. From the analysis of the student responses we concluded that the IPM teaching-learning model had advantages over the traditional mode of teaching and learning in two principal aspects. First, the integrating of the Maple animation with the PowerPoint presentation makes it much easier for instructors to explain certain concepts and methods and for students to understand the explanations in the classroom lectures. On the other hand, in traditional mode of lecture presentation, the instructor has to be an expert with artistic skills in graph sketching and had to spend a lot of time in drawing proper graphs to explain concepts and methods. With the Maple graphic function, the instructor can show students the figures from different angles and thus have more time to focus on concepts and methods with the graphic visualization aids. The second major advantage of the developed model is that it enhances students' self-learning capability for the subject. In the traditional mode of self-learning, those students lacking artistic skills found it extremely difficult to sketch the integration domain and subsequently determine the integration bounds even though they understand the concepts and methodology, while with the learning package, students can use the graphic function of Maple to draw the integration region easily and systematically even without any artistic skills. Also, through the exercises, students can gradually develop the skills of graph sketching. In addition, the learning package can provide solution in symbolic form which can then be used to check the solution obtained manually.

\section{Conclusion}

An efficient computer aided mathematics teaching-learning model and tool for triple integral calculus has been developed to enhance students' interest in mathematics. It provides students with an opportunity to have self-motivation, self-planned learning and selfassessment. Teacher and students have more time to focus on more practical examples to cover a wider variety of problems in class. Interactive Maplets of triple integrals enable students to analyze mathematics concepts step by step and also to self assess their homework. Maplets provide fast solutions and good visualizations of applied mathematics problems which can help students to identify patterns and see connections. Students can change functions and the values of parameters during the process of learning to understand the influence of different parameters to the output critically. These allow students to deepen their knowledge of multivariable calculus.

The paper has also demonstrated step by step how to use the IPM teaching-learning model for teaching triple integral calculus. Many useful graphs and Maple animation objects are also given in the paper which can be used to assist in explanation of concepts and methods for the triple integral calculus. The paper has thus provided a useful guideline and resources for the teaching of multivariate integral calculus using modern computation technology and graphic visualization. 
Our study has also shown that the proper use of symbolic computation systems in mathematics teaching can improve students' learning outcomes. Buchberger (1989) introduced the "white-box/black box principle" in which the author advocates that the pertinent part of the symbolic computation systems should not be used in the "white-box" phase of teaching a particular mathematical topic (i.e, the phase in which the topic is new to the students), while it is essential to use these systems in the "black-box" phase (in which the students completely master the topics). Although the "white-box/black box principle" provides a good general guidance for the use of symbolic computation systems in mathematics teaching, our investigation shows that for multivariate integral calculus, it is also very useful to use symbolic computation systems, in particular the graphic visualization functions, in the white-box phase of teaching.

\section{References}

Beltzer, A. I., \& Shenkman, A. W. (1995). Use of symbolic computation in engineering education. IEEE Transactions on Education, 38(2), 177-184. doi:10.1109/13.387221

Buchberger, B. (1989). Should students learn integration rules? ACM SIGSAM Bulletin, 24, 10-17. doi:10.1145/382276.1095228

Cecil, D. R. (2002). Graphs and enhancing maple multiplication. Mathematics and Computer Education, 36(1), 62-69.

Cheung, Y. L. (1996). Learning number theory with a computer algebra system. International Journal of Mathematical Education in Science and Technology, 27(3), 379-385.

Cook, D. (2006). Maple graphing tools for calculus III. Mathematics and Computer Education, 40(1), 36-41.

Drijvers, P. (2000). Students encountering obstacles using CAS. International Journal of Computers for Mathematical Learning, 5, 189-209.

Drijvers, P. (2002). Learning mathematics in a computer algebra environment: obstacles are opportunities. ZDM - The International Journal on Mathematics Education, 34(5), 221228.

Fuchs, K. J. (2001). Computer algebra systems in mathematics education. ZDM - The International Journal on Mathematics Education, 35(1), 20-23.

Hayden, M. B. and Lamagna, E. A. (1998). Newton: An interactive environment for exploring mathematics. Journal of Symbolic Computation, 25, 195-212. doi:10.1006/jsco.1997.0172

Heid, M. (1988). Resequencing skills and concepts in applied calculus using the computer as a tool. Journal for Research in Mathematics Education, 19(1), 3-25. doi:10.2307/749108

Johnson, D., \& Buege, J. (1995). Rethinking the way we teach undergraduate physics and engineering with Mathematica. In V. Keranen, P. Mitic (Eds.), Mathematics with VisionProceedings of the First International Mathematica Symposium (pp.233-242). Southampton, England. 
Kendal, M., \& Stacey, K. (2002). Teacher in transition: Moving towards CAS-supported classroom. ZDM - The International Journal on Mathematics Education, 34(5), 196-201.

Kong, S. C., \& Kwok, L. F. (1999). An interactive teaching and learning environment for graph sketching. Computers \& Education, 32(1), 1-17. doi:10.1016/S03601315(98)00032-3

Kramarski, B., \& Hirsch, C. (2003). Using computer algebra systems in mathematical classrooms. Journal of Computer Assisted Learning, 19, 35-45. doi:10.1046/j.02664909.2003.00004.x

Lagrange, J. B. (1999). Complex calculators in the classroom: Theoretical and practical reflections on teaching pre-calculus. International Journal of Computers for Mathematical Learning, 4, 51-81.

Man, Y. K. (2007). Introducing computer algebra to school teachers of mathematics. Teaching Mathematics and Its Applications, 20(1), 23-26.

Mathews, J. H. (1990). Using a computer algebra system to teach double integration. International Journal of Mathematical Education in Science and Technology, 21(5), 723732. doi: 10.1080/0020739900210506

Noinang, S., Wiwatanapataphee, B., \& Wu, Y.H. (2008). Teaching-learning-tool for integral calculus. Proceedings of the $13^{\text {th }}$ Asian Technology Conference in Mathematics (pp.525533). Suan Sunandha Rajabhat University, Bangkok, Thailand.

Peck, R. (2008). Introduction to statistics and data analysis (3rd ed.). Belmont, CA: Thomson Brooks/Cole.

Perjési, I. H. (2003). Application of CAS for teaching of integral-transforming theorems. ZDM - The International Journal on Mathematics Education, 35(2). 43-47. doi:10.1007/BF02652771

Peschek, W., \& Schneider, E. (2002). CAS in general mathematics education. ZDM - The International Journal on Mathematics Education, 34(5), 189-195.

Røyrvik, O. (2002). Teaching electrical engineering using maple. International Journal of Electrical Engineering, 39(4), 297-300.

Stewart, J. (2003). "Triple integrals-Calculus (5 $5^{\text {th }}$ ed.). Belmont, California: Thomson/ Brooks/ Cole.

Tall, D. (1991). Recent developments in the use of the computer to visualize and symbolize calculus concepts. MAA Notes, 20, 15-25.

Tonkes, E. J., Loch, B. I., \& Stace, A. W. (2005). An innovation learning model for computation in first year mathematics. International Journal of Mathematical Education in Science and Technology, 36(7), 751-758.

Ward, J. P. (2003). Modern mathematics for engineers and scientists. Teaching Mathematics and Its Applications, 22(1), 37-44. 
Wu, Y. H., \& Wiwatanapataphee, B. (2008). Lecture note: Advanced calculus. Australia: Curtin University of Technology.

\section{Authors}

Benchawan Wiwatanapataphee, Department of Mathematics, Faculty of Science, Mahidol University, Bangkok 10400, Thailand; scbww@ mahidol.ac.th

Sakda Noinang, Department of Mathematics, Statistics and Computer, Faculty of Science, Ubon Rachathani University, Ubon Rachathani 34190, Thailand; s.noinang@gmail.com

Yong Hong Wu, Department of Mathematics and Statistics, Curtin University of Technology Perth, Western Australia 6845, Australia; yhwu@maths.curtin.edu.au

Buraskorn Nuntadilok, Department of Mathematics, Faculty of Science, Mahidol University, Bangkok 10400, Thailand; jopal_ex@hotmail.com 


\section{Appendix}

The following Maple procedures were used in this paper.

\section{Maple commands for Figure $1 \mathrm{~b}$}

> \# 1. Procedure for generating sub-boxes.

$>$ with(plots): with(plottools):

$>$ CreateBoxes $:=\operatorname{proc}(x, y, z)$

local $i, j, k$;

global boxes;

for $i$ from 1 to $x$ do

for j from 1 to $y$ do

for $k$ from 1 to $z$ do

boxes $[i, j, k]:=\operatorname{display}(\operatorname{cuboid}([i-1, j-1, k-1],[i, j, k]$,

$$
\text { color=blue)) }
$$

od:

od:

od:

end proc:

$>$ \# 2. Procedure for accumulating all sub-boxes.

$>$ TripleRiemann $:=\operatorname{proc}(x, y, z)$

local $i, j, k$;

global SumBoxes;

SumBoxes $[0,1,1]:=$ boxes $[1,1,1]$ :

for $k$ from 1 to $z$ do

for $j$ from 1 to $y$ do

for $i$ from 1 to $x$ do

SumBoxes $[i, j, k]:=\operatorname{display}(\operatorname{SumBoxes}[i-1, j, k]$,

boxes $[i, j, k]$, color=blue);

SumBoxes $[0, j+1, k]:=$ SumBoxes $[i, j, k]$;

SumBoxes $[0,1, k+1]:=$ SumBoxes $[i, j, k]$;

od:

od:

od:

end proc:

$>x:=4: y:=6: z:=4$ :

$>$ CreateBoxes $(x, y, z)$ :

$>\operatorname{TripleRiemann}(x, y, z)$ :

$>$ \# 3. Command to display animation a list of figures.

$>\operatorname{display}([\operatorname{seq}(\operatorname{seq}(\operatorname{seq}(\operatorname{SumBoxes}[i, j, k], i=1 . . x), j=1 . . y), k=1 . . z)]$, axes $=$ normal, insequence $=$ true, scaling $=$ constrained, 
view $=[0 . . x, 0 . . y, 0 . . z]$, tickmarks $=[[x=" l "],[y=" m "],[z=" n "]])$;

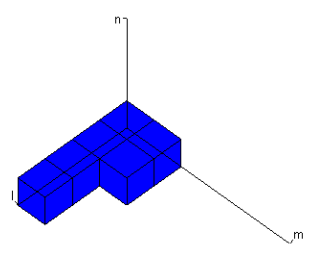

(a)

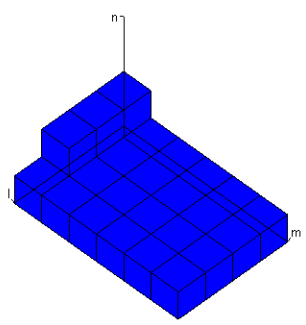

(b)

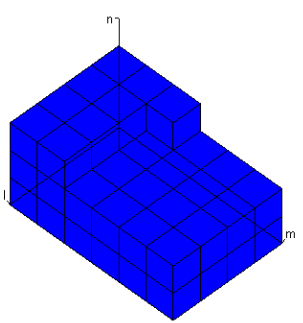

(c)

Figure 1b

\section{Maple commands for Figure 2b}

$>$ with(plots):

$>$ PiValue:=evalf(Pi):

$>y \min :=5$ :

$>$ ymax $:=y \min +3 *$ PiValue:

$>$ ymid $:=y \min +3 *$ PiValue $/ 2$ :

$>$ \# 1. Functions to determine boundary shape on xy-plane

$>f:=y->\cos ((y-5)-$ PiValue $/ 2)+5:$

$>g:=y->-\operatorname{sqrt}\left((3 * \text { PiValue } / 2)^{\wedge} 2-((y-5)-3 * \text { PiValue } / 2)^{\wedge} 2\right)+5$ :

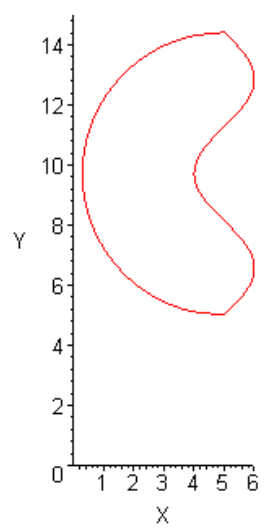

Figure 2b(a)

$>$ \# 2. Functions to determine 3D surfaces of upper and lower boundaries.

$>$ zUpper: $=(x, y)->18-(((x)-\text { PiValue }) / 3)^{\wedge} 2-((y-10) / 3)^{\wedge} 2:$

$>$ Lower: $=(x, y)->(((x)-\text { PiValue }) / 3)^{\wedge} 2+((y-10) / 3)^{\wedge} 2:$

$>$ PlotzUpper $:=\operatorname{plot} 3 d(z \operatorname{Upper}(x, y), x=0 . .2 *$ PiValue,$y=y$ min-

.5..ymax+.58):

$>$ PlotzLower $:=\operatorname{plot} 3 d(z$ Lower $(x, y), x=0 . .2 *$ PiValue,$y=y$ min-

$.5 . . y \max +.58)$ : 
$>$ \# 3. Procedure for generating a list of points $(x, y)$ on the boundary.

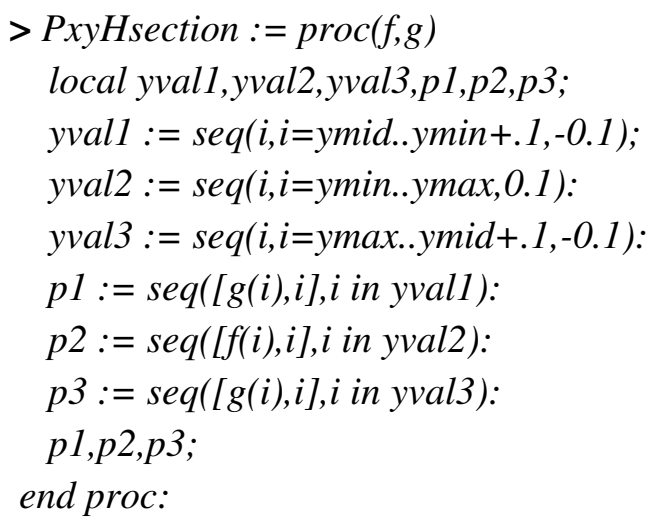

$>$ \# 4. Procedure for projecting the boundary as shown in Figure 2-b(a) to upper and lower $3 D$ surfaces obtained from step2.

$>$ PxyzUpperPart $:=\operatorname{proc}(L, t)$

local $u$;

[seq([u[1],u[2],t], $u=L)],[\operatorname{seq}([u[1], u[2], z \operatorname{Upper}(u[1], u[2])], \quad u=L)]$; end proc:

$>$ PxyzLowerPart $:=\operatorname{proc}(L, t)$

local $u$;

[seq([u[1],u[2],zLower(u[1],u[2])], $u=L)],[\operatorname{seq}([u[1], u[2], t], \quad u=L)]$; end proc:

$>$ PxyzSurfaceAll $:=\operatorname{proc}(L)$

local $u$;

[seq([u[1],u[2],zLower(u[1],u[2])], u=L)],[seq([u[1],u[2],

$z \operatorname{Upper}(u[1], u[2])], u=L)]$;

end proc:

> \# 5. Procedure for generating 3-D points on the boundary as shown in Figure 2-b(a) at $z=t$.

$>$ PxyzHsection $:=\operatorname{proc}(L, t)$

local $u$;

[seq $([u[1], u[2], t], u=L)]$;

end proc:

> \# 6. Procedure for initializing a list of points to generate any body surface.

$>$ PxyBody $:=\operatorname{proc}(L 1, L 2)$

local $i, n$;

$n:=\operatorname{nops}(L 1)$;

$\operatorname{seq}([L 1[i], L 2[i], L 2[i+1], L 1[i+1]], i=1 . . n-1)$

,[L1[n],L2[n],L2[1],L1[1]];

end proc: 
$>$ \# 7. Functions for initializing two lists of points to generate boundary surfaces

from $z=k$ to $z=z$ Upper and from $z=z$ Lower to $z=k$, respectively.

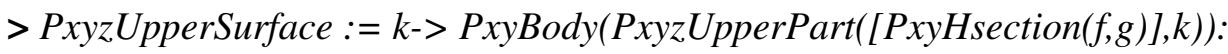

$>$ PxyzLowerSurface $:=k->$ PxyBody $($ PxyzLowerPart $([P x y H \operatorname{section}(f, g)], k))$ :

> \# 8. Procedures and build-in command 'spacecurve' for generating 3-D points of projected images boundary on upper and lower surfaces.

$>$ PxyzBLineUpSur $:=\operatorname{proc}(L)$

local $u$;

[seq([u[1],u[2],zUpper(u[1],u[2])], u=L)];

end proc:

$>$ PxyzBLineLoSur $:=\operatorname{proc}(L)$

local $u$;

[seq([u[1],u[2],zLower(u[1],u[2])], $u=L)]$;

end proc:

$>$ BLineUpSur $:=$ PxyzBLineUpSur $([$ PxyHsection $(f, g)])$ :

$>$ BLineLoSur $:=$ PxyzBLineLoSur $([$ PxyHsection $(f, g)])$ :

$>$ BLineUpper $:=$ spacecurve $($ BLineUpSur,color=black, thickness=2):

$>$ BLineLower $:=$ spacecurve $($ BLineLoSur,color=black,thickness $=2)$ :

$>$ \# 9. Three functions to generate surface.

$>$ UpperSurface $:=k->$

PLOT3D(seq(POLYGONS(b),b=PxyzUpperSurface $(k))$, STYLE(PATCHNOGRID),COLOR(X YSHADING)):

$>$ LowerSurface $:=k->$

PLOT3D(seq(POLYGONS(b),b=PxyzLowerSurface( $k)), \operatorname{STYLE}($ PATCHNOGRID),COLOR(X YSHADING)):

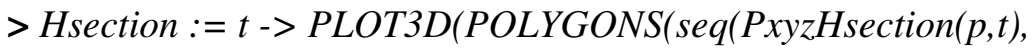

$p=[[$ PxyHsection $(f, g)]])), S C A L I N G(C O N S T R A I N E D), S T Y L E(P A T C H N O G R I D))$ :

$>$ \# 10. Using build-in command 'PLOT3D' and three functions as defined in Step 9 to generate surface.

$>$ SurfaceAll :=

PLOT3D(seq(POLYGONS(b),b=PxyBody(PxyzSurfaceAll([PxyHsection $(f, g)]))), \operatorname{STYLE}(P A T$ CHNOGRID),COLOR(XYSHADING)):

$>$ FigO := display(PlotzUpper,PlotzLower,BLineUpper,BLineLower,Hsection(-2)):

$>$ for i from 1 to 5 do

Fig $[i]:=\operatorname{display}($ Fig0,UpperSurface(15-i),LowerSurface(3+i),Hsection(15-

i),Hsection $(3+i))$

end do:

$>$ Fig6 := display $($ FigO,SurfaceAll): 
$>$ \# 14. Using build-in function 'display' to animate a list of figures.

$>$ display ([Fig0,seq(Fig[i],i=1..5),Fig6],insequence $=$ true, axes $=$ normal, orientation $=[46,57]$, , abels $=\left[X^{\prime}, Y^{\prime}, Z^{\prime}\right]$, view $=[-3 . .20,-8 . .15,-3 . .20]$,scaling=constrained, lightmodel=light 1$)$;

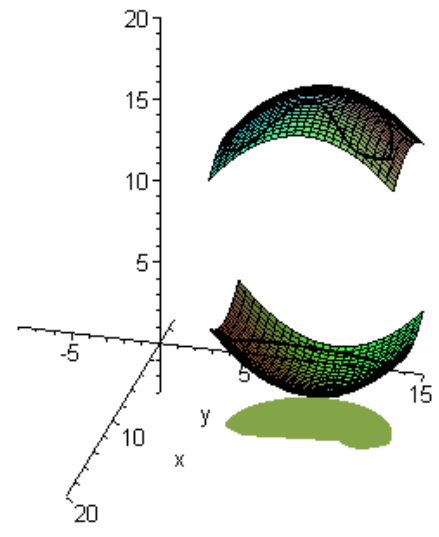

(b)

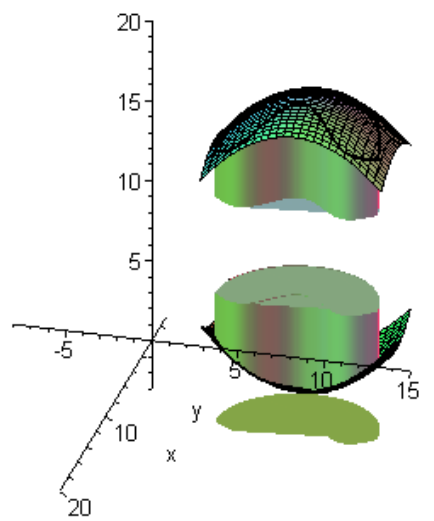

(c)

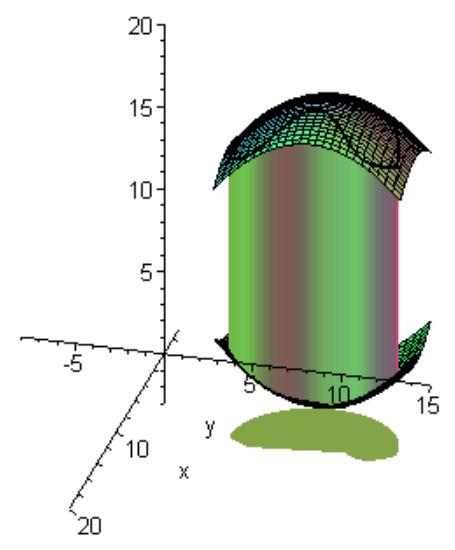

(d)

Figure $2 b$

\section{Maple commands for Figure 3a}

> \# 1. Using the following build-in commands to generate 5 figures: Fig1 - Fig5.

$>$ with(plottools): with(plots):

$>$ Line $1:=$ line $([0,0,0],[1,1,1]$, linestyle $=1$, thickness $=3$, color $=$ red $)$ :

$>$ Line $2:=$ line $([0,0,0],[1,1,0]$,thickness $=3$, color=green $)$ :

$>$ Line3 $:=$ line $([1,1,0],[1,1,1]$, thickness $=3$, color $=$ yellow $)$ :

$>$ LineX $:=$ line $([1,0,0],[1,1,0]$, linestyle $=7$, color=black $):$

$>$ LineY $:=$ line $([0,1,0],[1,1,0]$, linestyle $=7$, color $=$ black $)$ :

$>$ LineZ $:=$ line $([0,0,1],[1,1,1]$, linestyle $=7$, color=black $)$ :

$>$ Point $1:=\operatorname{point}([1,1,1]$, color $=$ green, symbol=CIRCLE):

$>$ Text $1:=$ textplot $3 d\left(\left[1.1,1.1,1.1,{ }^{\prime} p(r\right.\right.$, theta,$\left.z) '\right]$, color $=b l u e$, font=[TIMES, ROMAN, 14]):

$>$ Text $2:=$ textplot $3 d\left(\left[.5, .5,0.05,{ }^{\prime}{ }^{\prime}\right]\right.$, color $=$ red, font=[TIMES,ROMAN,14]):

$>$ Text $3:=$ textplot3d $\left(\left[1.1,1.1, .5,{ }^{\prime} z^{\prime}\right]\right.$, color $=$ red, font $=[T I M E S$, ROMAN,14]):

$>$ Text $X:=$ textplot $3 d\left(\left[1,-0.1,0, x^{\prime}\right]\right.$, color $=$ brown, font $=[T I M E S$, ROMAN,14]):

$>$ Text $Y:=$ textplot $3 d\left(\left[-0.1,1,0,{ }^{\prime} y^{\prime}\right]\right.$, color $=$ brown, font=[TIMES,ROMAN,14]):

$>$ TextZ $:=$ textplot $3 d\left(\left[-0.1,-0.1,1,{ }^{\prime} z^{\prime}\right]\right.$, color $=$ brown, font $=[T I M E S, R O M A N, 14]):$

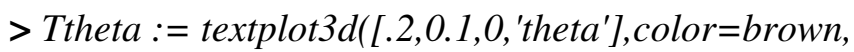
font=[TIMES, ROMAN, 14]): 
$>$ cylin $:=\operatorname{plot} 3 d([\operatorname{sqrt}(2) * \cos ($ theta $), \operatorname{sqrt}(2) * \sin ($ theta $), z]$,

theta $=0 . .2 * P i, z=0 . .1 .2$, shading $=x y$, axes $=$ normal,

orientation $=[-2,50]$, style $=$ patchnogrid, transparency $=.5)$ :

$>$ CylinCir $:=\operatorname{plot} 3 d([\operatorname{sqrt}(2) * \cos ($ theta $), \operatorname{sqrt}(2) * \sin ($ theta $), z]$,

theta $=0 . .2 * P i, z=0.995 . .1$, color $=$ black $)$ :

$>$ ThetaPlane $:=\operatorname{plot} 3 d([t, t, z], t=0 . . s q r t(2), z=0 . .1 .2$,

style $=$ patchnogrid, color $=$ red $)$ :

$>$ zplane $:=\operatorname{plot} 3 d([x, y, 1], x=-1.5 . .1 .5, y=-1.5 . .1 .5$, style $=$ wireframe $):$

$>$ angle $:=\operatorname{plot} 3 d([0.4 * r * \cos ($ theta $), 0.4 * r * \sin ($ theta $), 0]$,

theta $=0 . . P i / 4, r=1 . .1 .0001$, color $=r e d)$ :

$>$ struc $:=$ plots[display](Line1,Line2,Line3,LineX,LineY,LineZ,Point1, axes $=$ normal, orientation $=[-2,50])$ :

$>$ Fig1 $:=$ display $\left(\right.$ cylin,title $=$ "Cylinder radius $\left.r^{\prime \prime}\right)$ :

$>$ Fig2 $:=$ display (cylin,ThetaPlane,title="Vertical plane which make angle theta with $x$ axis"):

$>$ Fig3 $:=$ display $($ cylin,ThetaPlane, ,plane,title $=$ " plane $Z=z^{\prime \prime}$, CylinCir):

$>$ Fig4 := display(cylin,ThetaPlane,zplane,struc,Text2,Text3,TextX, TextY,TextZ,Ttheta, angle,title="The Cylindrical coordinates",CylinCir):

$>$ Fig5 := display(struc,Text1,Text2,Text3,TextX,TextY,TextZ,

Ttheta, angle,title="The Cylindrical coordinates"):

> \# 2. Using build-in function 'display' to animate a list of figures.

> display([Fig1,Fig2,Fig3,Fig4,Fig5],insequence=true);

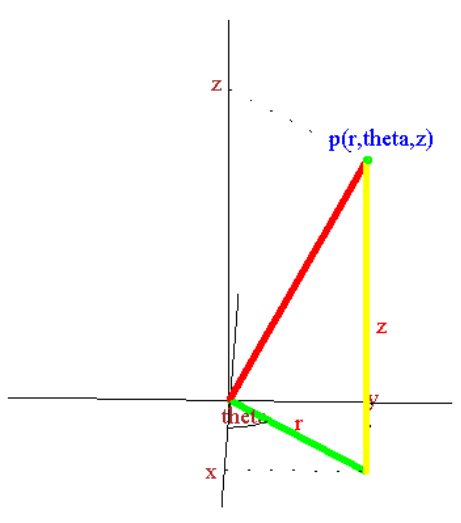

(a)

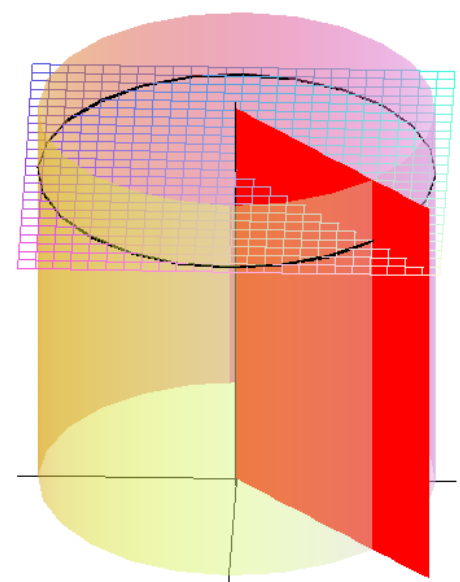

(b)

Figure 3a

\section{Maple commands for Figure 3b}


$>$ with(plots): with(plottools):

$>$ \# 1. Functions to generate cylinders of radius $k$ without and with wireframe style.

$>$ CylinRadiusk $:=k->$

plot $3 d([k * \cos ($ theta $), k * \sin ($ theta $), z]$, theta $=0 . .2 * P i, z=0 . .4$, scaling $=$ constrained, shading $=x y$, ax

es $=$ normal, orientation $=[77,51]$, view $=[-2 . .2,-2 . .2,0 . .4 .5]$, grid $=[24,6])$ :

$>$ CylinRadiuskWire $:=k->$

$\operatorname{plot} 3 d\left(\left[k^{*} \cos (\right.\right.$ theta $), k^{*} \sin ($ theta $\left.), z\right]$, theta $=0 . .2 * P i, z=0 . .4$, style $=$ wireframe, scaling $=$ constrain ed, shading $=x y$, color $=$ blue, axes $=$ normal, orientation $=[77,51]$, view $=[-2 . .2,-$

2..2,0..4.5], grid=[24,6]):

$>$ \# 2. Functions to display a part of cylinder tube.

$>$ PartCylinOutside $:=($ zbegin,zend $)->$

plot $3 d([2 * \cos ($ theta $), 2 * \sin ($ theta $), z]$, theta $=3 * P i / 4 . . P i, z=z$ begin..zend, scaling=constrained,sh ading $=x y$, axes $=$ normal):

$>$ PartCylinInside $:=($ zbegin,zend $)->$

plot3d([cos(theta), sin(theta),z],theta $=3 * P i / 4 . . P i, z=z$ begin..zend, scaling=constrained, shading $=x y$, axes $=$ normal):

$>$ \# 3. Functions to display a disc and a part of disc cylinder tube.

$>$ ZPlaneDisk $:=(z$,begin_r $)->$

plot $3 d\left(\left[r^{*} \cos (\right.\right.$ theta $), r^{*} \sin ($ theta $\left.), z\right], r=$ begin_r..2,theta $=0 . .2 *$ Pi,style $=$ patch,axes $=$ normal, gri $d=[6,24])$ :

$>$ PartOfZPlaneDisk $:=z->$

plot $3 d\left(\left[r^{*} \cos (\right.\right.$ theta $), r * \sin ($ theta $\left.), z\right], r=1 . .2$, theta $=3 *$ Pi/4..Pi,style= patchnogrid,axes $=$ normal $)$ :

$>$ \# 4. Using built-in function 'plot3d' to display a vertical plane and a part of a vertical plane.

$>$ Plane $1:=\operatorname{plot} 3 d([-t, t, z], t=0 . .2, z=0 . .4$, style=patchnogrid $)$ :

$>$ Plane $2:=\operatorname{plot} 3 d([t, 0, z], t=-2.5 . .0, z=0 . .4$, style $=$ patchnogrid $):$

$>$ PartOfPlane $1:=\operatorname{plot} 3 d([-t, t, z], t=\operatorname{sqrt}(2) / 2 . . \operatorname{sqrt}(2), z=1 . .2):$

$>$ PartOfPlane $:=\operatorname{plot} 3 d([t, 0, z], t=-2 . .-1, z=1 . .2)$ :

$>$ \# 5. Using built-in function 'line' and 'textplot3d' to generate lines as shown in Figure 3-

$b(a)$ and $(c)$ and texts as shown in Figure 3-b(a).

$>$ line $1:=$ line $([0,0,4],[0,2,4]$,thickness $=50$, color=blue $)$ :

$>$ line $2:=$ line $([0,0,4],[-\operatorname{sqrt}(2) / 2, \operatorname{sqrt}(2) / 2,4]$, thickness $=50$, color=red):

$>T 1:=$ textplot $3 d\left(\left[0,1.5,4.1,{ }^{\prime} r=r 1^{\prime}\right]\right.$, color $=$ blue,font=[TIMES, ROMAN,14] $):$

$>T 2:=$ textplot3d([-0.5,0.5,4.1,'r=r2'],color=red,font=[TIMES,ROMAN,14]):

$>l 1:=$ line $([0,0,1],[-1,0,1]$, color $=$ blue $)$ :

$>l 2:=$ line $([0,0,1],[-\operatorname{sqrt}(2) / 2, \operatorname{sqrt}(2) / 2,1]$, color $=$ blue $):$

$>l 3:=$ line $([0,0,2],[-1,0,2]$, color $=$ blue $):$

$>l 4:=$ line $([0,0,2],[-\operatorname{sqrt}(2) / 2, \operatorname{sqrt}(2) / 2,2]$, color=blue $):$

> \# 6. Constructing 6 figures for animation. 
$>$ Figl $:=$

display(CylinRadiusk(2),ZPlaneDisk(0,0),ZPlaneDisk(4,0), line1,T1,title= "Cylinder radius $\left.r l^{\prime \prime}\right)$ :

$>$ Fig2 :=

display(CylinRadiusk(2),CylinRadiusk(1),ZPlaneDisk(0,1),ZPlaneDisk(4,1),line1,T1,line2,T2

,title $=$ "Cylinders radius $r 1$ and $r 2 ")$ :

$>$ Fig $3:=$

display(CylinRadiuskWire(2),CylinRadiusk(1),PartCylinOutside(0,4),PartOfZPlaneDisk(0),P artOfZPlaneDisk(4), Plane1, Plane2):

$>$ Fig4 :=

display(CylinRadiuskWire(2),CylinRadiusk(1),PartCylinOutside(0,2),ZPlaneDisk(2,1),Plane

1,Plane2):

$>$ Fig $5:=$

display(CylinRadiuskWire(2),CylinRadiusk(1),PartCylinOutside(1,2),ZPlaneDisk(1,1),ZPlan eDisk(2,1),Plane1,Plane2):

$>$ Fig6 :=

display(PartCylinInside(1,2),PartCylinOutside(1,2),PartOfZPlaneDisk(1),PartOfZPlaneDisk (2),PartOfPlane1,PartOfPlane2,l1,l2,13,l4, orientation=[77,51],view=[-2..2,-2..2,0..4.5]):

> \# 7. Using build-in function 'display' to animate a list of figures.

$>$ display([Fig1,Fig2,Fig3,Fig4,Fig5,Fig6],insequence =true, orientation=[77,51],view=[-

$2 . .2,-2 . .2,0 . .4 .5]$,tickmarks $=[0,0,0])$;

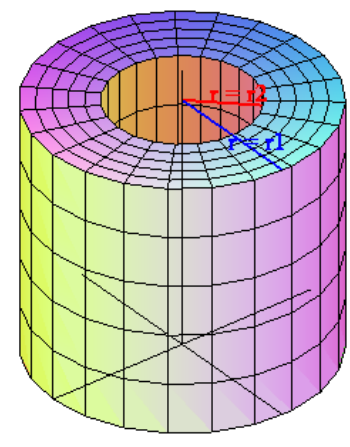

(a)

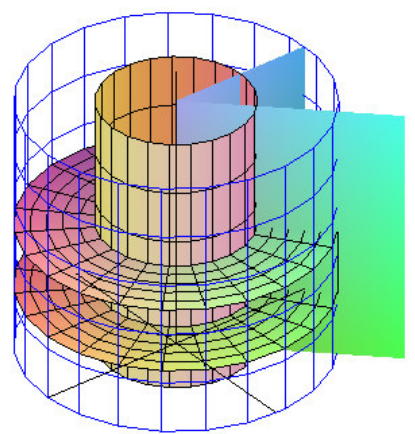

(b)

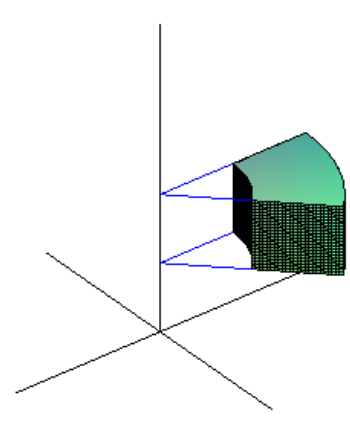

(c)

Figure $3 b$

\section{Maple commands for Figure 5a}

$>$ with(plots): with(plottools):

> \# 1 Using the following build-in commands to generate 5 figures: Fig1 - Fig5.

$>$ Line $1:=$ line $([0,0,0],[1,1,1]$,linestyle $=1$, thickness $=50$, color $=$ red $)$ :

$>$ Line $:=$ line $([0,0,0],[1,1,0]$, thickness $=50$, color $=$ green $):$

$>$ Line $:=$ line $([1,1,0],[1,1,1]$, thickness $=50$, color $=$ yellow $)$ :

$>$ LineX $:=$ line $([1,0,0],[1,1,0]$, linestyle $=7$, color=black $)$ : 
$>$ LineY $:=$ line $([0,1,0],[1,1,0]$, linestyle $=7$, color $=$ black $):$

$>$ LineZ $:=$ line $([0,0,1],[1,1,1]$,linestyle $=7$, color $=$ black $)$ :

$>$ PointRhoThetaPhi $:=\operatorname{point}([1,1,1]$, color $=$ green, symbol=CIRCLE):

$>$ TextRhoThetaPhi:=

textplot3d([1.1,1.1,1.1,'p(rho,theta,phi)'],color=blue,font=[TIMES,ROMAN,14]):

$>$ TextRho $:=$ textplot3d([.5,.5,.5,'rho'],color=red,font=[TIMES,ROMAN, 14]):

$>\operatorname{Text} X:=\operatorname{textplot} 3 d\left(\left[1,-0.1,0, x^{\prime}\right]\right.$, color=brown,font=[TIMES,ROMAN,14]):

$>$ TextY $:=$ textplot $3 d\left(\left[-0.1,1,0, y^{\prime}\right]\right.$, color=brown,font=[TIMES,ROMAN,14]):

$>$ TextZ $:=$ textplot3d $\left(\left[-0.1,-0.1,1,{ }^{\prime} z^{\prime}\right]\right.$, color=brown,font $\left.=[T I M E S, R O M A N, 14]\right)$ :

$>$ TextTheta $:=$ textplot $3 d\left(\left[.2,0.1,0,{ }^{\prime}\right.\right.$ theta'], color=brown,font=[TIMES,ROMAN,14]):

TextPhi $:=$ textplot3d([.1,0.1,0.2,'phi'],color=brown,font=[TIMES, ROMAN,14]):

$>$ AngleTheta $:=$

plot $3 d([0.4 * r * \cos ($ theta $), 0.4 * r * \sin ($ theta $), 0]$, theta $=0 .$. Pi/4, $r=1 . .1 .0001$, color $=$ red $)$ :

AngleRho :=

plot $3 d([0.4 * r * \sin ($ theta $), 0.4 * r * \sin ($ theta $), 0.4 * r * \cos ($ theta $)]$, theta $=0 .$. Pi/4, $r=1 . .1 .0001$, color $=$ red):

$>$ struc : $=$

plots[display](Line1,Line2,Line3,LineX,LineY,LineZ,PointRhoThetaPhi,axes=normal,orienta tion $=[9,73])$ :

$>$ SphereWireframe: $=$

plot $3 d([\operatorname{sqrt}(3) * \sin ($ phi $) * \cos ($ theta $), \operatorname{sqrt}(3) * \sin ($ phi $) * \sin ($ theta $), \operatorname{sqrt}(3) * \cos ($ phi $)]$, phi $=0 . . P i$, th eta $=0 . .2 *$ Pi,scaling $=$ constrained, shading $=x y$, axes $=$ normal, style $=$ wireframe $)$ :

$>$ SpherePlaneIntersecLine: $=$

plot3d([rho* $\sin ($ phi $) * \cos (P i / 4), r h o * \sin (p h i) * \sin (P i / 4), r h o * \cos (p h i)], p h i=0 . . P i, r h o=1.72 . . s q r$ t(3), color=black):

$>$ ConeInSphere :=

plot $3 d([r h o * \sin (P i / 3.25) * \cos ($ theta $), r h o * \sin (P i / 3.25) * \sin ($ theta $), r h o * \cos (P i / 3.25)]$, rho $=0 . . s q$ $r t(3)$, theta $=0 . .2 * P i$,scaling $=$ constrained, shading $=x y$, axes $=$ normal, style = patchnogrid $)$ :

$>$ plane $1:=\operatorname{plot} 3 d([t, t, z], t=0 . . \operatorname{sqrt}(2), z=-\operatorname{sqrt}(3) . . \operatorname{sqrt}(3)$, style=patchnogrid $)$ :

$>$ Fig1 $:=\operatorname{display}($ SphereWireframe, orientation $=[9,73])$ :

$>$ Fig2 := display(plane 1,SphereWireframe,orientation=[9,73],SpherePlaneIntersecLine):

$>$ Fig3 :=

display(ConeInSphere,plane 1,SphereWireframe, orientation=[9,73],SpherePlaneIntersecLine ,Line1):

$>$ Fig4 :=

display(struc,AngleTheta,AngleRho,TextPhi,ConeInSphere,plane1,SphereWireframe,SphereP laneIntersecLine):

$>$ Fig $5:=$

display(struc,TextRhoThetaPhi,TextRho,TextX,TextY,TextZ,TextTheta,AngleTheta,AngleRho, TextPhi):

> \# 2. Using build-in function 'display' to animate a list of figures.

$>$ display([Fig1,Fig2,Fig3,Fig4,Fig5],insequence=true); 


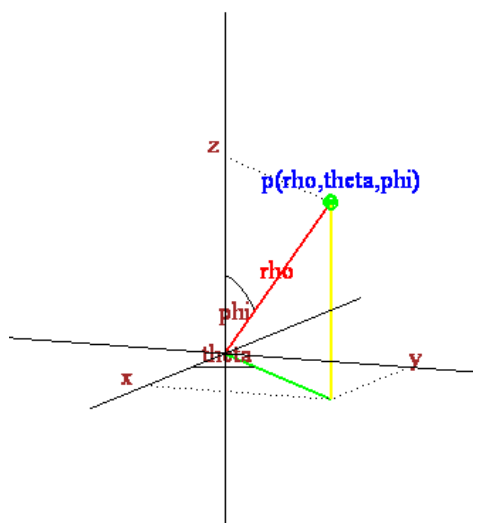

(a)

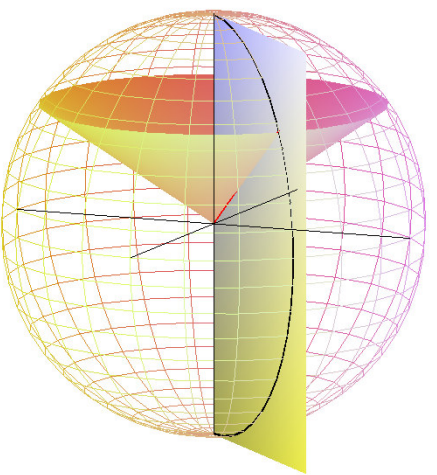

(b)

Figure 5a

\section{Maple commands for Figure 5b}

$>$ with(plots): with(plottools):

$>$ \# 1. Using build-in command 'plot3d' to generate inside and outside sphere with and without wireframe style.

> InsideSphere: $=$

plot3d $([\sin (p h i) * \cos ($ theta $), \sin ($ phi $) * \sin ($ theta $), \cos ($ phi $)]$, phi $=0 .$. Pi, theta $=0 . .2 * P i$, scaling $=$ co nstrained, shading $=x y$, axes $=$ normal, view $=[-2 . .2,-2 . .2,-2 . .2])$ :

$>$ OutsideSphere: $=$

plot $3 d([1.5 * \sin ($ phi $) * \cos ($ theta $), 1.5 * \sin ($ phi $) * \sin ($ theta $), 1.5 * \cos ($ phi $)]$, phi $=0 . . P i$, theta $=0 . .2 * P$ $i$, scaling $=$ constrained, shading $=x y$, axes $=$ normal, view $=[-2 . .2,-2 . .2,-2 . .2])$ :

$>$ InsideSphereWire: $=$ plot $3 d([\sin ($ phi $) * \cos ($ theta $), \sin ($ phi $) * \sin ($ theta $), \cos ($ phi $)]$, phi $=0 .$. Pi, theta $=0 . .2 *$ Pi, scaling $=$ co nstrained, , hading $=x y$, axes $=$ normal, view $=[-2 . .2,-2 . .2,-2 . .2]$, style $=$ wireframe, color $=$ blue $)$ :

$>$ OutsideSphereWire: $=$ plot $3 d([1.5 * \sin ($ phi $) * \cos ($ theta $), 1.5 * \sin ($ phi $) * \sin ($ theta $), 1.5 * \cos ($ phi $)]$, phi $=0 .$. Pi, theta $=0 . .2 * P$ $i$, scaling $=$ constrained, shading $=x y$, axes $=$ normal, view $=[-2 . .2,-2 . .2,-2 . .2]$, style $=$ wireframe $)$ :

> \# 2. Functions to display a part of inside and outside sphere.

$>$ PartOfInsideSphere $:=($ phibegin,phiend $)->$ plot $3 d([\sin ($ phi $) * \cos ($ theta $), \sin ($ phi $) * \sin ($ theta $), \cos ($ phi $)]$, phi=phibegin..phiend, theta $=P i / 6 . . P i$ 13 , scaling $=$ constrained, color $=$ blue, lightmodel $=$ light 2 , axes $=$ normal, view $=[-2 . .2,-2 . .2,-$

2..2], style=patchnogrid):

$>$ PartOfOutsideSphere: $=($ phibegin,phiend $)->$ plot $3 d([1.5 * \sin ($ phi $) * \cos ($ theta $), 1.5 * \sin ($ phi $) * \sin ($ theta $), 1.5 * \cos ($ phi $)]$, phi=phibegin..phiend,t heta $=$ Pi/6..Pi/3, scaling $=$ constrained, color $=$ red, lightmodel $=$ light, axes $=$ normal, view $=[-$ $2 . .2,-2 . .2,-2 . .2]$, style $=$ patchnogrid): 
$>$ \# 3. Using build-in command 'plot3d' to display two vertical planes and a part of planes.

$>$ plane $1:=\operatorname{plot} 3 d([t, 1 / \operatorname{sqrt}(3) * t, z], t=0 . .2, z=-2 . .2$, style $=$ patchnogrid $):$

$>$ plane $2:=\operatorname{plot} 3 d([t, \operatorname{sqrt}(3) * t, z], t=0 . .2, z=-2 . .2$, style $=$ patchnogrid $):$

$>$ PartOfPlane $1:=$

plot3d $([r h o * \sin (p h i) * \cos (P i / 6), r h o * \sin (p h i) * \sin (P i / 6), r h o * \cos (p h i)], r h o=1 . .1 .5, p h i=P i / 6 . . P i /$

3 , scaling $=$ constrained, shading $=x y$, axes $=$ normal, view $=[-2 . .2,-2 . .2,-2 . .2])$ :

$>$ PartOfPlane $2:=$

plot3d $([r h o * \sin (p h i) * \cos (\mathrm{Pi} / 3), r h o * \sin (p h i) * \sin (\mathrm{Pi} / 3), r h o * \cos (p h i)], r h o=1 . .1 .5, \mathrm{phi}=\mathrm{Pi} / 6 . . \mathrm{Pi} /$

3 , scaling $=$ constrained, , $h$ ading $=x y$, axes $=$ normal, view $=[-2 . .2,-2 . .2,-2 . .2])$ :

$>$ PartOfPlane $3:=$

plot $3 d([r h o * \sin (P i / 6) * \cos ($ theta $), r h o * \sin (P i / 6) * \sin ($ theta $), r h o * \cos (P i / 6)]$, rho $=1 . .1 .5$, theta $=P$

$i / 6 . . P i / 3$, scaling $=$ constrained, shading $=x y$, axes $=$ normal, view $=[-2 . .2,-2 . .2,-2 . .2])$ :

$>$ PartOfPlane $4:=$

plot3d $([r h o * \sin (P i / 3) * \cos ($ theta $), r h o * \sin (P i / 3) * \sin ($ theta $)$, rho $* \cos (P i / 3)]$, rho $=1 . .1 .5$, theta $=P$

$i / 6 . . P i / 3$, scaling $=$ constrained, shading $=x y$, axes $=$ normal, view $=[-2 . .2,-2 . .2,-2 . .2])$ :

$>$ \# 4. Function to generate cross-section between a sphere and a plane.

$>$ SphereThetaPlaneIntersecLine: $=$ theta->

plot3d $([r h o * \sin ($ phi $) * \cos ($ theta $), r h o * \sin (p h i) * \sin ($ theta $), r h o * \cos ($ phi $)]$, phi=0..Pi,rho=1.49..1.

5$, scaling $=$ constrained, color $=$ black, axes $=$ normal, view $=[-2 . .2,-2 . .2,-2 . .2])$ :

> \# 5. Using build-in command 'plot3d' to display two cones.

$>$ cone $1:=$

plot $3 d([r h o * \sin (\mathrm{Pi} / 6) * \cos ($ theta $), r h o * \sin (\mathrm{Pi} / 6) * \sin ($ theta $)$, rho $* \cos (\mathrm{Pi} / 6)]$, rho $=0 . .2$, theta $=0 . .2$

$* P i$, scaling $=$ constrained, shading $=x y$, axes $=$ normal, view $=[-2 . .2,-2 . .2,-$

2..2], style=patchnogrid):

$>$ cone $2:=$

plot3d $\left(\left[r_{\text {rho }} * \sin (\mathrm{Pi} / 3) * \cos (\right.\right.$ theta $), r h o * \sin (\mathrm{Pi} / 3) * \sin ($ theta $)$, rho $\left.* \cos (\mathrm{Pi} / 3)\right]$, rho $=0 . .2$, theta $=0 . .2$

$*$ Pi, scaling $=$ constrained, shading $=x y$, axes $=$ normal, view $=[-2 . .2,-2 . .2,-$

2..2], style=patchnogrid):

> \# 6. Using built-in function 'line' to generate lines as shown in Figure 5-b(c).

$>$ Line $1:=\operatorname{line}([0,0,0],[\sin (\mathrm{Pi} / 3) * \cos (\mathrm{Pi} / 3), \sin (\mathrm{Pi} / 3) * \sin (\mathrm{Pi} / 3), \cos (\mathrm{Pi} / 3)]$, color=blue $):$

$>$ Line $2:=$ line $([0,0,0],[\sin (\mathrm{Pi} / 3) * \cos (\mathrm{Pi} / 6), \sin (\mathrm{Pi} / 3) * \sin (\mathrm{Pi} / 6), \cos (\mathrm{Pi} / 3)]$, color=blue $):$

$>$ Line $3:=\operatorname{line}([0,0,0],[\sin (\mathrm{Pi} / 6) * \cos (\mathrm{Pi} / 3), \sin (\mathrm{Pi} / 6) * \sin (\mathrm{Pi} / 3), \cos (\mathrm{Pi} / 6)]$, color=blue $):$

$>$ Line4 $:=$ line $([0,0,0],[\sin (\mathrm{Pi} / 6) * \cos (\mathrm{Pi} / 6), \sin (\mathrm{Pi} / 6) * \sin (\mathrm{Pi} / 6), \cos (\mathrm{Pi} / 6)]$, color=blue $):$

$>$ \# 7. Constructing 8 figures for animation.

$>$ Fig1 := display (OutsideSphereWire,title= "Sphere radius rhol"):

$>$ Fig2 : = display (OutsideSphereWire,InsideSphere,title="Spheres radius rho1 and rho2"):

$>$ Fig $3:=$

display(OutsideSphereWire,InsideSphere,plane2,SphereThetaPlaneIntersecLine(Pi/3),title=" Spheres are cut with plane1"): 
$>$ Fig $4:=$

display(OutsideSphereWire,InsideSphereWire,plane1,plane2,PartOfInsideSphere(0,Pi),Part OfOutsideSphere(O,Pi),SphereThetaPlaneIntersecLine(Pi/6),SphereThetaPlaneIntersecLine( $\mathrm{Pi} / 3)$,title="Spheres are cut with plane1 and plane2"):

$>$ Fig $5:=$ display(OutsideSphereWire,InsideSphereWire,plane 1,plane2,cone 1,PartOfInsideSphere(Pi/6, Pi),PartOfOutsideSphere(Pi/6,Pi),SphereThetaPlaneIntersecLine(Pi/6),SphereThetaPlaneInt ersecLine(Pi/3), title= "Spheres are cut with cone1"):

$>$ Fig6 : = display(OutsideSphereWire,InsideSphereWire,plane1,plane2,cone1,cone2,PartOfInsideSpher e(Pi/6,Pi/3),PartOfOutsideSphere(Pi/6,Pi/3),SphereThetaPlaneIntersecLine(Pi/6),SphereThe taPlaneIntersecLine(Pi/3), title = "Spheres are cut with cone1 and cone2"):

$>$ Fig $7:=$ display(InsideSphereWire,OutsideSphereWire,PartOfInsideSphere(Pi/6,Pi/3),PartOfOutsideS phere(Pi/6,Pi/3),PartOfPlane1,PartOfPlane2,PartOfPlane3,PartOfPlane4):

$>$ Fig8:= display(PartOfInsideSphere(Pi/6,Pi/3),PartOfOutsideSphere(Pi/6,Pi/3),PartOfPlane1,PartOf Plane2,PartOfPlane3,PartOfPlane4,Line 1,Line2,Line3,Line4):

> \# 8. Using build-in function 'display' to animate a list of figures.

$>$ display ([Fig1,Fig2,Fig3,Fig4,Fig5,Fig6,Fig7,Fig8], insequence=true, orientation=[38,77],ti ckmarks=[0,0,0]);

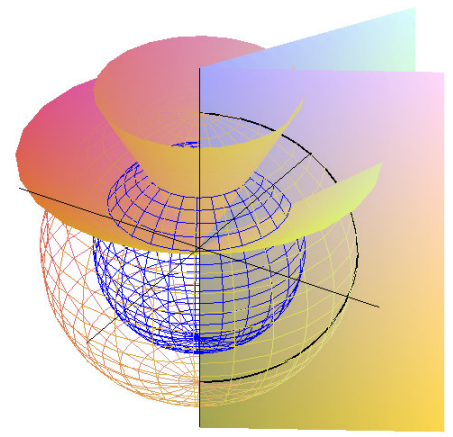

(a)

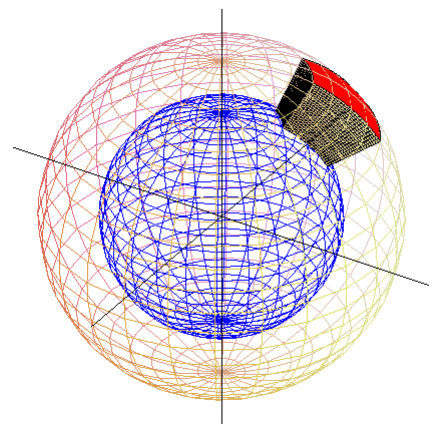

(b)

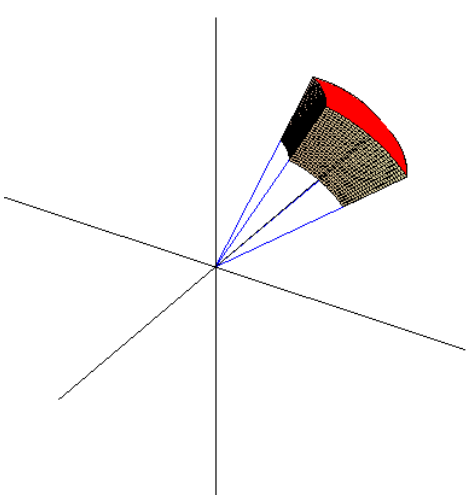

(c)

Figure $5 b$ 